\title{
NUEVOS REGISTROS DEL GENERO TRISETUM PERS. (POACEAE : AVENEAE) PARA LA FLORA DE CHILE
}

\author{
NEW RECORDS OF GENUS TRISETUM PERS. (POACEAE : AVENEAE) \\ FOR THE FLORA OF CHILE
}

\author{
Víctor L. Finot
}

RESUMEN

Se da a conocer la presencia de tres nuevos taxa del género Trisetum Pers. (Poaceae : Aveneae) para la flora de Chile, considerados hasta ahora endémicos de Argentina: $T$. ambiguum Rúgolo et Nicora, T. caudulatum Trin. var. correae Nicora y $T$. oreophilum Louis-Marie var. johnstonii Louis-Marie. Se incluye una descripción de cada taxón y un mapa de su distribución en Chile.

Palabras Claves: Trisetum, Poaceae, Nuevos

Registros, Flora de Chile.

\section{INTRODUCCION}

En Sudamérica, el género Trisetum Pers., comprende unas 25 especies que se distribuyen a lo largo de la Región Andina sudamericana, que comprende las altas cordilleras de Venezuela, Colombia y Ecuador, Perú, Bolivia, Chile y Argentina (Morrone 1999). La Patagonia chilenoargentina constituye un importante centro de diversificación específica del género, concentrando la mayor parte de las especies sudamericanas (Nicora

${ }^{1}$ Departamento de Producción Animal, Facultad de Agronomía, Universidad de Concepción, Casilla 537, Chillán, Chile.
The presence of three new taxa of genus Trisetum Pers. (Poaceae: Aveneae), until now considered endemic to Argentina, are reported for the Chilean flora: T. ambiguum Rúgolo et Nicora, T. caudulatum Trin. var. correae Nicora and T. oreophilum Louis-Marie var. johnstonii LouisMarie. A description for each taxon and a map of their distribution in Chile are included.

Keywords: Trisetum, Poaceae, New Records, Flora of Chile.

1978). Algunas de las especies presentes en esta región viven tanto en Chile como en Argentina, mientras que otras especies se conocen sólo de uno $\mathrm{u}$ otro territorio.

Zuloaga et al. (1994) señalan 16 especies y 3 variedades de Trisetum para la flora agrostológica argentina. De estos 19 taxa, 6 son reconocidos como endémicos: T. ambiguum Rúgolo et Nicora, $T$. caudulatum Trin. var. correae Nicora, T. cumingii (Nees ex Steud.) Nicora var. santacrucense Nicora, T. longiglume Hack. var. glabratum Nicora, $T$. oreophilum Louis-Marie var. johnstonii Louis-Marie y $T$. sclerophyllum Hack.

Para la flora de Chile, Marticorena \& Quezada (1985) citan 24 taxa (17 especies y 7 variedades). Posteriormente, Marticorena (1990) eleva a 25 el 
número de taxa presentes en Chile, con 18 especies y 7 variedades en Chile continental, una de las cuales (T. caudulatum Trin.), se encuentra también en el territorio insular Archipiélago de Juan Fernández (Marticorena et al. 1998).

Como parte de la revisión del género Trisetum para América, hemos podido establecer la presencia en Chile de tres taxa, hasta ahora considerados endémicos de Argentina. Ellos son T. ambiguum Rúgolo et Nicora, T. caudulatum Trin. var. correae Nicora y T. oreophilum Louis-Marie var. johnstonii Louis-Marie.

\section{MATERIALES Y METODOS}

Este estudio forma parte de la revisión taxonómica del género Trisetum en América. Los nuevos registros dados a conocer, se obtuvieron de la revisión de material de los siguientes herbarios: BA, BAA, BAB, CONC, LP, MERL, SGO, SI, USL, US y herb. Zoellner y tipos sudamericanos y fototipos del United States National Herbarium, Smithsonian Institution, Washington (US). Se utilizó, además, la base de datos del herbario CONC, que contiene la lista de taxa, colectores y localidades de las colecciones chilenas del género. Para las abreviaturas de los herbarios se siguió a Holmgren et al. (1990), con excepción del herbario del Prof. O. Zoellner (herb. Zoellner).

\section{RESULTADOS}

Trisetum ambiguum Rúgolo et Nicora, Bol. Soc. Argent. Bot. 25(3-4): 468, fig. 2. 1988. TIPO: Argentina, Prov. Santa Cruz, Dpto. Güer Aike, Ea. Sofía, Secc. Cuadrado, m/m $5 \mathrm{Km}$ al S de Estancia Punta del Monte, 350 m., 12-II-1978, 51ํ1's, $71^{\circ} 18^{\prime} \mathrm{W}$, T.B.P.A. 3119 (holotipo: BAB; isotipo: SI, no vistos).

Perenne, con cortos rizomas, $12-34 \mathrm{~cm}$ de alto. Culmo algo piloso bajo la panícula. Hojas cortas, no superando $1 / 2$ de la altura de la planta. Vainas inferiores glabras, las superiores pubescentes. Láminas planas, de 2-3 mm de ancho, convolutas, glabras. Panícula espiciforme a subespiciforme. Espiguillas 1-floras, raro 2-floras. Glumas iguales o la gluma superior levemente mayor y más ancha que la gluma inferior, agudas, con la quilla lisa o escasamente escabrosa. Gluma inferior de 5-7,5 mm, igual o poco menor que su antecio, 1-nervada. Gluma superior de 6-8 mm, mayor que su antecio o más raramente igual que él, 3-nervada. Antecio de 4-6 mm. Lemma escabrosa, 5-nervada, los nervios notables hacia el ápice. Apice de la lemma con 2

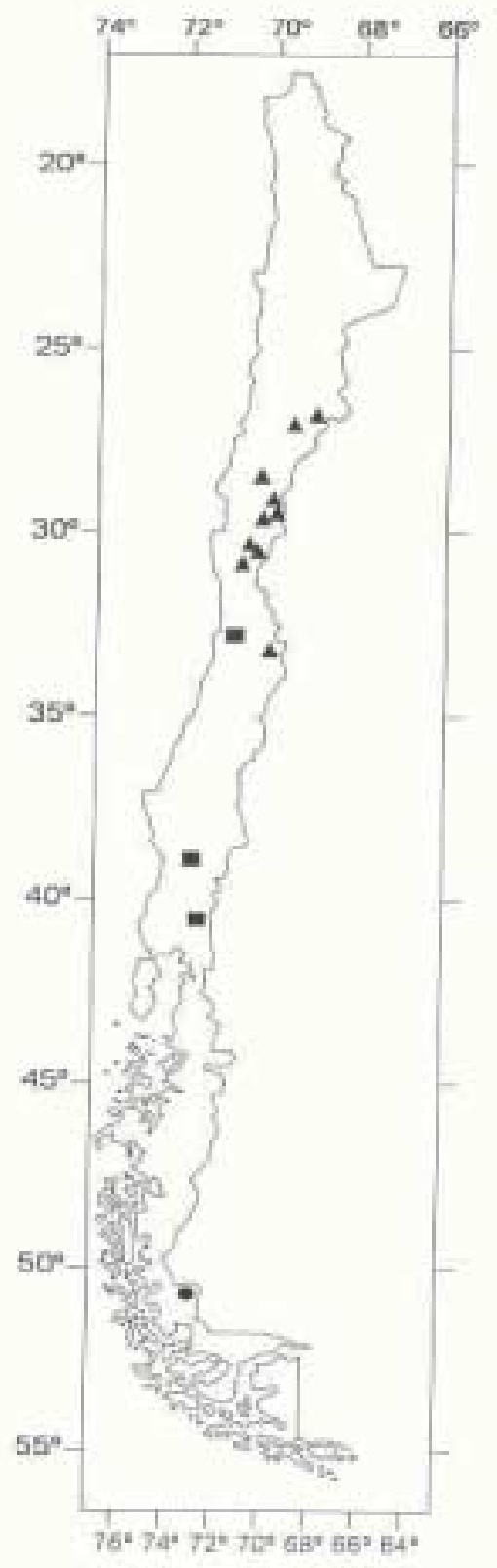

Figura 1. Distribución de Trisetum ambiguum Rúgolo \& Nicora (•), T. caudulatum Trin. var. correae Nicora ( $\square)$ y T. oreophilum Louis-Marie var. johnstonii Louis-Marie $(\boldsymbol{\Delta})$, en Chile. 
arístulas mayores y 2 menores. Arista fuertemente retorcida. Callus con pelos de ca. 0,5 $\mathrm{mm}$ de largo. Pálea membranosa, 2-nervada, menor que su lemma. Lodículas 2, hialinas, bilobuladas, de ca. $1 \mathrm{~mm}$. Estambres 3, anteras de $1 \mathrm{~mm}$ de largo. Cariopsis con 1 a varios pelitos curvos y brillantes en el ápice del rostro. Endosperma líquido.

ObSERVACIONEs: Morfológicamente, T. ambiguum es afín a $T$. cumingii, de la que se diferencia por sus espiguillas usualmente unifloras, sus glumas subisomorfas, generalmente mayores que los antecios y sus aristas varias veces geniculadas. En T. cumingii las glumas son desiguales, la inferior menor que la espiguilla, la superior mayor que ella.

Especie patagónica, fue dada a conocer por Rúgolo de Agrasar \& Nicora (1988) sobre la base de material colectado en las provincias de Santa Cruz y Tierra del Fuego, Argentina, al sur del paralelo $50^{\circ} \mathrm{S}$. En Chile ha sido colectada en la XII Región, Prov. de Ultima Esperanza, también al sur de los $50^{\circ} \mathrm{S}$ (Figura 1).

\section{Material Estudiado}

ARGENTINA, Prov. Santa Cruz, Dpto. Güer Aike, Valle Superior Río Turbio, mallín a $580 \mathrm{~m}$, 51'29'S, 7207'W, 6-II-1978, AMBROSETTI \& MÉNDEZ 29883 (TBPA 3857) (MERL); Valle

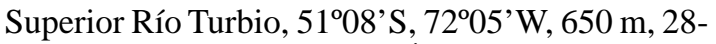
I-1978, AMBROSETTI \& MÉNDEZ 29875 (TBPA 3321) (MERL); Estancia La Carlota, sección San Elías, 57²4'S, 71³2'W, 19-I-1978, Roig, Anchorena, MÉNDEZ \& AMBROSETTI 103 (TBPA-FIT 2783 a) (SI); Prov. Tierra del Fuego, Norte de Río Grande, Estancia María Behety, 27-I1955, SORIANO 4847 (BAA); Cerca cerro Mesa, 31-I-1942, CASTELLANOS s.n. (BA 45577); Castillo, 30-I-1942, CASTELLANOS s.n. (BA 45560); Río Grande, 1-III-1917, BONARELLI s.n. (SI 12037).

CHILE, XII Región, Prov. de Ultima Esperanza, Sierra de los Baguales, Estancia La Cumbre, Campo de La Tropilla, 5044'S, 72²2' W, 900 m, 29-I-1987, LANDERO 790 (CONC 93158); Sierra de los Baguales, Cerro Santa Lucía, 5044'S, 72²2'W, $900 \mathrm{~m}, 15-\mathrm{I}-1985$, ARROYO 85160 (CONC 120266); Sierra del Toro, 51 ${ }^{\circ} 10^{\prime}$ 'S, 7250'W, 700-750 m, 9-II-1992, ARROYO, VON BOHLEN,
GARCÍA \& GIGOUX 9276 (CONC 128423); 10II-1992, ARROYO, VON BOHLEN, GARCÍA \& GIGOUX 92112 (CONC 155197).

Trisetum caudulatum Trin. var. correae Nicora, Fl. Patag. 3: 254. fig. 164e. 1978. TIPO: Argentina, Prov. Chubut, Dpto. Futaleufú, lago Futalaufquén, Soriano 4334 (holotipo: BAA visto).

Perenne, $35 \mathrm{~cm}$ de alto, con rizomas estoloniformes. Vainas menores que los entrenudos. Culmo glabro. Vainas y láminas pilosas, excepcionalmente glabras y ciliadas en el margen. Láminas planas, 2-4 mm de ancho. Lígula $\mathrm{m} / \mathrm{m}$ truncada, 3-4 mm long. Panícula de 6-15 x 1,5-4 cm, contraída a espiciforme, densiflora, interrumpida o no, con ramas inferiores de $2-3 \mathrm{~cm}$, a veces con tintes violáceos. Raquis escabroso. Espiguilla 2-4(-5)-flora, de hasta $8 \mathrm{~mm}$, abiertas en el ápice. Pedicelos 1-3 $\mathrm{mm}$, escabrosos. Glumas desiguales, ambas menores que los antecios, aristuladas en el ápice, raro la superior iguala a su antecio. Gluma inferior de 4-6 x 0,3 mm, 1-3-nervada, linear-lanceolada, atenuada. Gluma superior de 5-8 x $1 \mathrm{~mm}, 3$-5-nervada, oval-lanceolada. Antecio inferior de 7,5 x $0.7 \mathrm{~mm}$, los superiores menores. Raquilla de $1 \mathrm{~mm}$ de largo, con pelos de hasta 1,5 mm. Callus con pelos cortos, de ca. 0,5 mm. Lemma glabra, algo escabrosa, linear-lanceolada, con los márgenes membranosos aproximados dejando la pálea libre de la lemma. Apice de la lemma biaristulado, arístulas de hasta $1 \mathrm{~mm}$. Arista inserta en el 1/3 o 1/4 superior. Lodículas de ca. 0,6 mm, bilobuladas en el ápice, los lóbulos agudos. Pálea de $6 \mathrm{~mm}$ en el antecio inferior, hialina, 2-nervada, los nervios escabrosos, el ápice 2-aristulado por prolongación de los nervios. Lodículas de $1 \mathrm{~mm}, 2-$ lobuladas en el ápice. Estambres 3, anteras de 1,2 mm. Ovario y cariopsis con pelitos curvos y brillantes en el ápice. Cariopsis rostrada, de 4 x 0,7 mm. Endosperma líquido.

OBSERVACIONES: Este taxón se distingue de la variedad típica por la presencia de pelitos en el ápice del ovario y de la cariopsis. Nicora (1978) describió esta variedad de Trisetum caudulatum Trin., sobre la base de material colectado en las Provincias de Neuquén, Río Negro y Chubut, Argentina. Probablemente, las primeras colecciones de este taxón en Chile corresponden a las realizadas por Rodulfo Amando Philippi en la X Región en 1877. En Chile vive entre las regiones V y X, al igual que la variedad típica (Figura 1). 
Material Estudiado

ARGENTINA, Prov. Neuquén, Dpto. Los Lagos, $3 \mathrm{~km}$ W de Confluencia, 20-XII-1982, GONZÁLEZ 766 (LP); Lago Traful Sur, orilla del lago, 18-I-1973, RÚGOLO DE AGRASAR 233 (BAA 12443); P.N. Nahuel Huapí, Lago Trafuel, El Mirador, 7-XI-1949, BOELCKE \& HUNZIKER 3657 (BAA); Prov. Río Negro, P.N. Nuahuel Huapí, campamento Río Villegas, 29-I-1941, PÉREZ MOREAU s.n. (BA 37679); P.N. Nuahuel Huapí, faldeos cerro Santa Elena, 31-I-1952, FABRIS \& SOLBRIG 1171 (LP 890202); Prov. Chubut, P.N. Los Alerces, Lago Futalafquén, entre Río Desaguadero y Arroyo del Salto, 12-XII-1962, LAHITTE \& ROQUERO 277 (BA); Lago Futalaufquén, 7-I-1964, Lahitte s.n. (BA).

CHILE, V Región, Punta Imán, Cerro Roble, 2000 m, 8-XII-1991, Zoellner 18196 (herb. ZOELLNER); IX Región, Valle de Lonquimay,

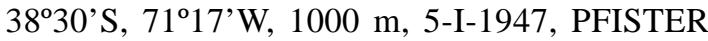
S.N. (CONC 7256); X Región, Lago Llanquihue, sin fecha, PHILIPPI s.n. (SGO 45835); Posada del Valle, I-1877, PHILIPPI s.n. (SGO 45834).

Trisetum oreophilum Louis-Marie var. johnstonii Louis-Marie, Rhodora 30: 237. 1929. TIPO: Argentina, Andes of N.W. San Juan, Arroyo Tambillos, 4300 m, 29¹0'S, 6951'W, 10-I-1926, Johnston 6097 (holotipo: GH; isotipos: US 1297379, SGO 59037).

Perenne, cespitosa, 10-20 cm de alto, 1-nodes. Culmo glabro o piloso bajo la panícula. Vainas y láminas pilosas. Vaina superior $\mathrm{m} / \mathrm{m}$ inflada $\mathrm{y}$ estriada. Láminas basales de 3-8 cm de largo x 11,5 mm de ancho, planas o conduplicadas, angostas. Lámina suprema de $1-5 \mathrm{~cm}$ x ca. $1 \mathrm{~mm}$, conduplicada. Lígula 0,5-1 $\mathrm{mm}$, con el margen mediano truncado, dentado-ciliado. Panícula oval, espiciforme, interrumpida en la base, densa, verde y purpúrea, 2-4,5 x 0,8-1,5 cm. Raquis glabro o piloso. Espiguillas de 3,5-4,5 mm, 2-3 floras, subsésiles o con pedicelos de hasta $3 \mathrm{~mm}$, escabrosos. Glumas menores que los antecios o la superior los iguala, anchas, subiguales, escabrosas en la quilla, agudas en el ápice. Gluma inferior de 2,7-4 x 0,4-0,9 mm, 1 (-3) nervada, los nervios laterales cuando presentes, muy cortos, no alcanzan 1/2 del cuerpo de la gluma. Gluma superior de 3,34,7 x 0,5-1,2 mm, 3-nervada. Antecio inferior de
3,5-4 x 0,7 mm, los superiores menores. Lemma teñida de púrpura en el ápice, éste biaristulado por la prolongación de los nervios medianos de la lemma, hirsuta, cubierta de pelos tiesos y cortos hasta el nacimiento de la arista inserta en el tercio superior. Arista 2-3,5 mm, algo retorcida en la base y geniculada o curvada, escabrosa. Callus obtuso, con pelos cortos, ca. $0,5 \mathrm{~mm}$. Raquilla densamente pilosa, de ca. $1 \mathrm{~mm}$, con pelos de hasta 1,5 $\mathrm{mm}$ de largo. Pálea menor que la lemma en ambos antecios o subigual a la lemma en el antecio superior, 2,23,5 $\mathrm{mm}$ en el antecio inferior, hialina, 2-nervada, los nervios escabrosos, el ápice bidentado. Lodículas ca. $0,5 \mathrm{~mm}$, bilobadas en el ápice, hialinas. Anteras 1,1 mm long. Cariopsis 1,6-2 x 0,3-0,5 mm, glabra en el ápice. Endosperma líquido.

Observaciones: Taxón endémico de Sudamérica, habita en la Cordillera de Los Andes del norte de Chile y Argentina, entre los 3000 y los $4600 \mathrm{~m}$ de altitud. Fue originalmente descrita para la Argentina, norte de la Provincia de San Juan $\left(29^{\circ} \mathrm{S}\right)$, donde fue colectada por I.M. Johnston en 1926. Es posible que debido a su similitud morfológica con $T$. preslei E.Desv. su presencia haya pasado inadvertida en Chile, por lo que ha sido considerada, hasta ahora, una especie endémica de Argentina, de las provincias de San Juan y Mendoza, aproximadamente entre los 27 y $33^{\circ} \mathrm{S}$. (Zuloaga et al. 1994). Sobre la base de material revisado en los herbarios CONC, SGO y USL, hemos podido determinar su presencia en Chile, entre la III Región (aprox. $27^{\circ} \mathrm{S}$ ) y la Región Metropolitana $\left(33^{\circ} \mathrm{S}\right)$ (Figura 1).

\section{Material Estudiado}

ARGENTINA, Prov. San Juan, Espinacito, Los Frías, río Las Leñas, 3700 m, Ene-II-1949, ROIG 11950 (BAA); Dpto. Calingasta, entre Paso Espinacito Sur y Quebrada Honda,12-II-1950, PÉREZ-MOREAU \& PERRONE s.n. (BA 54919); Cordillera de Colanguil, quebrada del Salto, 15-I1930, PÉREZ-MOREAU 30-258 (BA, BAA); Prov. Mendoza, Dpto. San Rafael, Paso Cruz de Piedra, Refugio Perón, 3800 m, 16-I-1952, ROIG 50 (MERL 49482); San Carlos, camino a Laguna Diamante, mallines superiores a 3300 m, 3-II-1950, Boelcke 4132 1/2 (BAA); Laguna Diamante, 3320 m, 18-I-1963, Boelcke et al. 10039 (BAA); Laguna 
Diamante, El Paramillo, 3700 m, 18-I-1963, BOELCKE et al. 10061 (BAA); Laguna Diamante, 17-I-1963, BOELCKE et al. 10011 (BAA). Dpto. Luján, Lagunita del Plata, 4150 m, 23-II-1983, TROMBOTTO \& AHUMADA s.n.(MERL 53194). Cordillera Frontal, Cordón del Plata, Lagunita del Plata, $4600 \mathrm{~m}, 33^{\circ} 03^{\prime} \mathrm{S}$, 69²4'W, 19-22-II-1984, TROMBOTTO s.n.(MERL 37944).

CHILE, III Región, Prov. Huasco, Quebrada Cantarito, entre Quebrada Marancel y Portezuelo de Cantarito, 3500-4300 m, 28³9' S, 6943'W, 23-I-1983, MARTICORENA et al. 83462-B (CONC 55165); Prov. Copiapó, Laguna del Negro Francisco, 3520 m, 27²9' S, 69¹6'W, 2-II- 944, MUÑOZ 3981 (SGO 119711); km 42 río del Estrecho, 3950 m, 29¹7'S, 7004'W, 19I-1994, ARANCIO, SQUEO Y LEÓN 94250 (CONC 129177, USL 4437); Quebrada Los Barriales, 29¹5'S, 7003'W, 3900 m, 15-I-1994, ARANCIO, SQUEO Y LEÓN 94043 (USL 4402); IV Región, Prov. Limarí, Cordillera de Ovalle, Vegas San Miguel, $3000 \mathrm{~m}, 30^{\circ} 51^{\prime} \mathrm{S}$, 70³1'W, 13-I-1959, JILES 3649 (CONC 105500, SGO 73774); Prov. Elqui, Cordillera Doña Ana, Quebrada del Negro, $4250 \mathrm{~m}$, 2947'S, 7000'W, 18-II-1992, ARANCIO 92129 (CONC 122033, USL 2214); Cordillera de Doña Ana, Camino a Tambo, 2948' S, 695 ' $\mathrm{W}, 4300$ m, 18-II-1992, ARANCIO 92129 (USL 2213); Cordillera de Doña Ana, 23 Mar 1994, Arancio s.n. (USL); Cordillera de Doña Ana, 2951'S, 6955'W, 4250 m, 20-I-1993, ARANCIO 93021 (USL); Prov. Limarí, Cordillera de Ovalle, Doña Rosa, nacimiento de Quebrada Larga, 3750 m, 3044'S, 70²3'W, 10-I-1956, JILES 2952 (CONC 105498); Cordillera de Ovalle, Punta de Huana-río Molles, 3900 m, 3043'S, 70³1'W, 3-II-1962, JILES 4136 (CONC 101778, CONC 105492); Prov. Coquimbo, Cordillera de Ovalle, San Miguel-Los Pingos, $3500 \mathrm{~m}$, 3053' S, 70³4'W, 19-I-1972, JILES 5889 (CONC 101709); Cordillera de Ovalle, Los Pingos, faldeos pedregosos, $3500 \mathrm{~m}$, 3053' $\mathrm{S}$, 70³4' $\mathrm{W}$, 19-I-1972, JILES 5888 (CONC 102936); Prov. Choapa, Cordillera de Illapel, Caletón Blanco, $3000 \mathrm{~m}, 31^{\circ} 28^{\prime} \mathrm{S}, 70^{\circ} 40^{\prime} \mathrm{W}, \mathrm{C}$. JILES 4246 (CONC 105493). Cordillera de Combarbalá, Hacienda Ramadilla, 3300 m, 31 ${ }^{\circ} 18^{\prime} \mathrm{S}, 70^{\circ} 50^{\prime} \mathrm{W}$, 21-I-1966, JILES 4801 (CONC 105494, CONC
102940); Cajón de Los Pelambres, $3400 \mathrm{~m}$, 31 ${ }^{\circ} 45^{\prime}$ S, 70³0'W, 15-I-1989, TEILLIER 1536 (CONC 121066); Región Metropolitana, Prov. Santiago, Paso de las Nieves Negras, alta cordillera andina, $3300 \mathrm{~m}, 33^{\circ} 47^{\prime} \mathrm{S}, 6^{\circ} 54^{\prime} \mathrm{W}$, II1949, GUNCKEL 20465a (CONC 144989).

\section{AGRADECIMIENTOS}

Deseo expresar mi agradecimiento a los curadores de los Herbarios BA, BAA, BAB, CONC, MERL, LP, SGO, SI, US, USL y al Prof. Otto Zoellner, quienes pusieron el material a nuestra disposición, para su estudio. Al Prof. Fernando Zuloaga, por su estímulo y ayuda durante esta revisión y también por el envío de material del Herbario del Instituto Botánico Darwinion, San Isidro, Buenos Aires, Argentina. Al Prof. Clodomiro Marticorena, por su ayuda y revisión crítica del manuscrito. Al Prof. Oscar Matthei su constante guía e inestimable ayuda y revisión del manuscrito. A Alicia Marticorena, sus valiosas sugerencias. A los revisores anónimos, las valiosas correcciones hechas al manuscrito. Al Sr. Pedro Arias por la confección del mapa. A la Dirección de Investigación, Universidad de Concepción, Proyecto DIUC 201.121.005-1.0.

\section{BIBLIOGRAFIA}

Holmgren, P.K., N.H. Holmgren \& L.C. Barnett. 1990. Index herbariorum, Part 1. The herbaria of the world (ed. 8). Regnum Vegabile 120: 1693.

Marticorena, C. 1990. Contribución a la estadística de la flora vascular de Chile. Gayana, Bot. 47: 85-113.

Marticorena, C. Y M. Quezada. 1985. Catálogo de la flora vascular de Chile. Gayana, Bot. 42(12): 1-147.

Marticorena, C., T.F. Stuessy \& C.M. Baeza. 1998: Catalogue of the vascular flora of the Robinson Crusoe or Juan Fernández Islands, Chile. Gayana Bot. 55(2):187-211.

Morrone, J.J. 1999. Presentación preliminar de un nuevo esquema biogeográfico de América del Sur. Biogeographica 75: 116.

Nicora, E.G. 1978. Gramineae, en Correa, M.N. (ed.), Flora Patagónica 3: 1-563.

Rúgolo De Agrasar, Z.E. \& E.G. Nicora. 1988. 
Gayana Bot. 59(1), 2002

Nuevos taxones para la Argentina y Chile Austral (Gramineae). Bol. Soc. Argent. Bot. 25 (3-4): 463-476.

Zuloaga, F., E. Nora, Z.E. Rúgolo De Agrasar, O.
Morrone, J. Pensiero \& A.M. Cialdella. 1994. Catálogo de la familia Poaceae en la República Argentina. Monogr. Syst. Bot., Missouri Bot. Gard. 47: I-XII, 1-178.

Fecha de recepción: 15.04 .02

Fecha de aceptación: 10.05.02 